Pacific Journal of Mathematics

A REFORMULATION OF THE ARF INVARIANT ONE mod $p$
PROBLEM AND APPLICATIONS TO ATOMIC SPACES 


\title{
A REFORMULATION OF THE ARF INVARIANT ONE $\bmod p$ PROBLEM AND APPLICATIONS TO ATOMIC SPACES
}

\author{
PAul Selick
}

\begin{abstract}
A $(\bmod p)$ atomic space is one whose lowest nonvanishing $(\bmod p)$ homology group has dimension 1 and which has the property that all self-maps which induce isomorphisms on this lowest nonvanishing group are homotopy equivalences. An atomic space cannot be decomposed, up to homotopy, into a produce of other spaces and thus is, in some sense, an atom. In this paper we show that if $p$ is an odd prime and $n>1$ then $\Omega^{3} S^{2 n+1}$ and the homotopy-theoretic fibre of the double suspension $\Sigma^{2}$ : $S^{2 n-1} \rightarrow \Omega^{2} S^{2 n+1}$ are $(\bmod p)$ atomic. Some indecomposability results are also obtained for the homotopy-theoretic fibre of the degree $p$ map of $\Omega S^{2 n+1}$.
\end{abstract}

Introduction. In homotopy theory we can distinguish between the weak form of the Arf invariant problem which asks if a certain element in the Adams spectral sequence is an infinite cycle and the strong form which asks for a 3-cell complex with a nontrivial Bockstein and Steenrod operation. The strong form implies the weak form and it has been conjectured that they are equivalent. Ravenel's negative solution of the weak form of the problem for $p \geq 5$ shows (somewhat vacuously) that the conjecture holds in this case. (See [19].) If $p=2$, it has been shown that the weak form of the problem is equivalent to the Kervaire problem. (See Browder [4].) Barratt and Mahowald have shown that divisibility of a certain Whitehead product by 2 implies the weak form of the $(\bmod 2)$ Arf invariant problem. (See [15], Corollary 2.) In fact it is well-known that divisibility of this Whitehead product by 2 is equivalent to the strong form of the Arf invariant problem, although I have been unable to find all the details in the literature. $\S 1$ gives a proof of this equivalence and generalizes the result to odd primes. Throughout this paper, the term Arf invariant will refer to the strong form.

$\$ 2$ proves a technical theorem which gives a sufficient condition for a self-map of a space to be a homotopy equivalence. The main results of the paper are in $\S 3$ where the results of $\S \S 1$ and 2 are applied to show that certain spaces are atomic. In particular (after localizing at an odd prime $p$ ) 
we obtain the following:

COROLLARY 3.4. $\Omega S^{2 n+1}\{p\}$ is atomic for all $n$ such that $\pi_{2 n(p-1)-2}^{s}$ has no elements of Arf invariant $1 \bmod p\left(\right.$ where $S^{2 n+1}\{p\}$ is the homotopy-theoretic fibre of the pth power map $\left.p: S^{2 n+1} \rightarrow S^{2 n+1}\right)$.

\section{CoRollary 3.5. If $p \geq 5$ and $n \neq 1$ or $p$ then $\Omega S^{2 n+1}\{p\}$ is atomic.}

THEOREM 3.7. $C(n)$ is atomic for $n>1$ (where $C(n)$ is the homotopytheoretic fibre of the double suspension $\left.\Sigma^{2}: S^{2 n-1} \rightarrow \Omega^{2} S^{2 n+1}\right)$.

THEOREM 3.8. $\Omega^{3} S^{2 n+1}$ is atomic for $n>1$.

I. A reformulation of the Arf invariant one problem. Let $p$ be a prime. In this section $H_{*}(X)$ will denote $H_{*}(X ; Z / p Z)$ and all spaces and maps will be assumed to have been localized at $p$. Let $P^{n}(k)=$ $S^{n-1} \cup_{k} e^{n}, n \geq 2$, where $k: S^{n-1} \rightarrow S^{n-1}$ is of degree $k$. Homotopy with $Z / k Z$ coefficients is defined by $\pi_{n}(X ; Z / k Z) \equiv\left[P^{n}(k), X\right]$. Many of its properties can be found in [16]. If $g: X \rightarrow Y$, we let $C_{g}$ denote the homotopy-theoretic cofibre of $g$.

Given $f: S^{m-1} \rightarrow S^{0}$ in $\pi_{m-1}^{s}, m>1$, since $f$ is torsion we can extend $f$ to $\hat{f}: P^{m}\left(p^{r}\right) \rightarrow S^{0}$ for some $r$. Of course, $\hat{f}$ is not uniquely determined by $f$. We say that $\pi_{2 n(p-1)-2}^{s}$ has an element of (strong) Arf invariant $1 \bmod p$ if there exists $C_{\hat{f}}$ in which the Steenrod operation $P^{n}$ (respectively: $\mathrm{Sq}^{2 n}$ ) acts nontrivially, where $f \in \pi_{2 n(p-1)-2}^{s}$.

Let $C(n)$ denote the homotopy-theoretic fibre of $\Sigma^{2}: S^{2 n-1} \rightarrow$ $\Omega^{2} S^{2 n+1} . C(n)$ is $2 n p-4$ connected. Let $f$ denote the composite

$$
S^{2 n p-3} \rightarrow C(n) \rightarrow S^{2 n-1}
$$

where the first map is a generator of $\pi_{2 n p-3}(C(n)) \cong Z / p Z$. It is well known that $f=0 \Leftrightarrow$ there exists an element of Hopf invariant 1 in $\pi_{2 n(p-1)-1}^{s}$. (See [10], Proposition 5.4, p. 300.)

Note. Here, and elsewhere, "equals" means equals within the set of homotopy classes of maps.

Suppose that $n \neq 1$ and that in addition if $p=2, n \neq 2$ or 4 so that $f \neq 0$. Notice that if $p=2$ then $f=\left[\iota_{2 n-1}, \iota_{2 n-1}\right]$.

THEOREM 1.1. The following are equivalent:

(a) $f=p g$ for some $g$

(b) There exists $h: P^{2 n p-2}(p) \rightarrow \Omega^{2} S^{2 n+1}$ such that $h_{*} \neq 0$ on $H_{2 n p-2}$.

(c) $\pi_{2 n(p-1)-2}^{s}$ has an element of Arf invariant 1 . 
Proof. Let $J_{k}(X)$ denote the $k$ th stage of the James construction on $X$. That is, $J_{k}(X)=X^{k} / \sim$ where

$$
\left(x_{1}, \ldots, x_{j-1}, *, x_{j+1}, \ldots, x_{k}\right) \sim\left(x_{1}, \ldots, x_{j-1}, x_{j+1}, *, x_{j+2}, \ldots, x_{k}\right) .
$$

Let $b^{\prime}$ be the statement

$\left(\mathrm{b}^{\prime}\right)$ There exists $h: p^{2 n p-2}(p) \rightarrow \Omega J_{p-1}\left(S^{2 n}\right)$ such that $h_{*} \neq 0$ on $H_{2 n p-2}$.

$b \Leftrightarrow b^{\prime}:$ There is a fibration

$$
J_{p-1}\left(S^{2 n}\right) \rightarrow \Omega S^{2 n+1} \stackrel{H}{\rightarrow} \Omega S^{2 n p+1}
$$

due to James [13] for $p=2$ and Toda [23] for $p>2$. So the pair $\left(\Omega^{2} S^{2 n+1}, \Omega J_{p-1}\left(S^{2 n}\right)\right)$ is $2 n p-2$ connected and thus $\mathrm{b} \Leftrightarrow \mathrm{b}^{\prime}$.

$a \Rightarrow b^{\prime}:$ There is a fibration

$$
S^{2 n-1} \stackrel{i}{\rightarrow} \Omega J_{p-1}\left(S^{2 n}\right) \stackrel{T}{\rightarrow} \Omega S^{2 n p-1}
$$

due to James [13] for $p=2$ and Toda [23] for $p>2$. From the definition of $f$ the composite

$$
S^{2 n p-3} \stackrel{f}{\rightarrow} S^{2 n-1} \stackrel{\Sigma^{2}}{\rightarrow} \Omega^{2} S^{2 n+1}
$$

is null homotopic. Because of the connectivity of the pair $\left(\Omega^{2} S^{2 n+1}, \Omega J_{p-1}\left(S^{2 n}\right)\right)$ it follows that

$$
S^{2 n p-3} \stackrel{f}{\rightarrow} S^{2 n-1} \stackrel{i}{\rightarrow} \Omega J_{p-1}\left(S^{2 n}\right)
$$

is null homotopic. Therefore $p(i g)=i_{\#}(p g)=i_{\#}(f)=0$. Thus there exists $h: P^{2 n p-2}(p) \rightarrow \Omega J_{p-1}\left(S^{2 n}\right)$ such that

$$
\begin{array}{ccc}
S^{2 n p-3} & \stackrel{j}{\rightarrow} & P^{2 n p-2}(p) \\
\downarrow g & & \downarrow h \\
S^{2 n-1} & \stackrel{i}{\rightarrow} & \Omega J_{p-1}\left(S^{2 n}\right)
\end{array}
$$

is homotopy commutative. We must show that $T h \neq 0$.

Suppose to the contrary that $T h=0$. Then there exists $h^{\prime}: P^{2 n p-2}(p)$ $\rightarrow S^{2 n-1}$ such that $h=i a$. So $i(g-a j)=i g-i a j=i g-h j=0$. Thus $\Sigma^{2}(g-a j)=0$. But

$$
\operatorname{ker} \Sigma^{2}: \pi_{2 n p-3}\left(S^{2 n-1}\right) \rightarrow \pi_{2 n p-3}\left(\Omega^{2} S^{2 n+1}\right)
$$


is $Z / p Z$, generated by $f$. So $g-a j=\lambda f$ for some $\lambda$. Multiplying by $p$ gives $p g-p(h j)=p \lambda f=0$. Also, $p(a j)=0$ since multiplication by $p$ kills $\operatorname{Im} j^{\#}$. Therefore $p g=0$. But $p g=f$ and so we have a contradiction.

Thus $T h \neq 0$ in $\pi_{2 n p-2}\left(\Omega S^{2 n p-1} ; Z / p Z\right)$. It follows from the $\bmod p$ Hurewicz isomorphism (see Neisendorfer [16], Theorem 3.8) that $T_{*} h_{*} \neq 0$ on $H_{2 n p-2}$. Since $T_{*}$ is an isomorphism on $H_{2 n p-2}, h_{*} \neq 0$ on $H_{2 n p-2}$.

$\mathrm{b}^{\prime} \Rightarrow \mathrm{c}$ : Let $h^{\prime}: P^{2 n p-1}(p) \rightarrow J_{p-1}\left(S^{2 n}\right)$ be the adjoint of $h$.

$$
H^{q}\left(C_{h^{\prime}}\right)=\left\{\begin{array}{ll}
Z / p Z & q=2 k n, \quad k \leq p \\
Z / p Z & q=2 n p-1 \\
0 & \text { otherwise }
\end{array}\right\} .
$$

Let $x$ be a generator of $H^{2 n}\left(C_{h^{\prime}}\right)$. Then $x^{p-1} \neq 0$. We show $x^{p} \neq 0$.

The map of homotopy-theoretic fibrations

$$
\begin{aligned}
& \Omega J_{p-1}\left(S^{2 n}\right) \quad \rightarrow \quad E J_{p-1}\left(S^{2 n}\right) \quad \rightarrow \quad J_{p-1}\left(S^{2 n}\right) \\
& \downarrow \Omega \gamma \quad \downarrow \quad \downarrow \gamma \\
& \Omega C_{h^{\prime}} \quad \rightarrow \quad E C_{h^{\prime}} \quad \rightarrow \quad C_{h^{\prime}}
\end{aligned}
$$

induces a map of cohomology Serre spectral sequences.
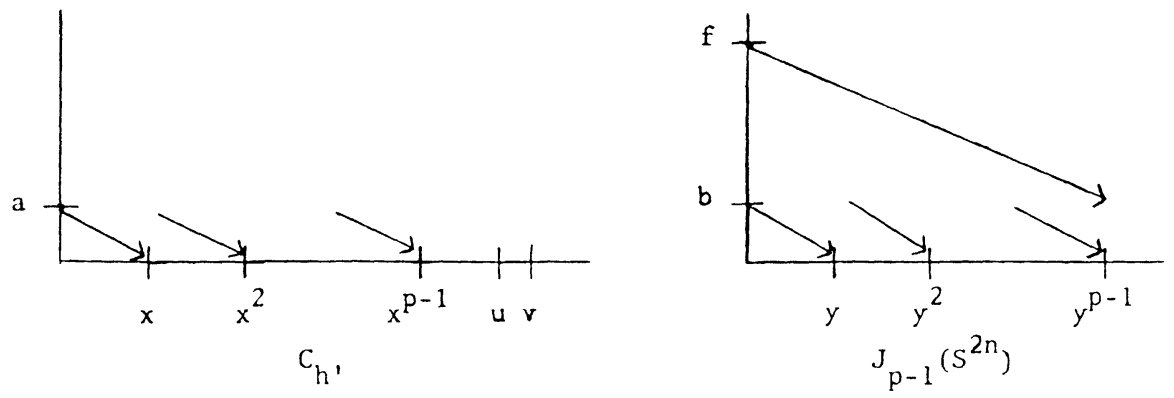

$d\left(x^{p-1} \otimes a\right)=x^{p}$. If $x^{p}=0$ then there exists $e \in H^{2 n p-2}\left(C_{h^{\prime}}\right)$ such that $d(e)=x^{p-1} \otimes a$. But then diagram chasing shows that $(\Omega \gamma)^{*}(e)=f$ so $(\Omega \gamma)^{*}$ is surjective on $H^{2 n p-2}$. Therefore $(\Omega \gamma)_{*}$ is injective on $H_{2 n p-2}$. So $(\Omega \gamma)_{*} h_{*} \neq 0$ on $H_{2 n p-2}$. But $(\Omega \gamma) h$ factors as

$$
P^{2 n p-2}(p) \rightarrow \Omega P^{2 n p-1}(p) \stackrel{\Omega h^{\prime}}{\rightarrow} \Omega J_{p-1}\left(S^{2 n}\right) \stackrel{\Omega \gamma}{\rightarrow} \Omega C_{h^{\prime}}
$$

and $\gamma h^{\prime}=0$. From this contradiction, we conclude $x^{p} \neq 0$. So $P^{n} x=x^{p}$ $\neq 0$.

Let $w$ be the composite

$$
P^{2 n p}(p) \stackrel{\Sigma h^{\prime}}{\rightarrow} \Sigma J_{p-1}\left(S^{2 n}\right) \rightarrow S^{2 n+1}
$$


where the second map is the adjoint of $J_{p-1}\left(S^{2 n}\right) \rightarrow \Omega S^{2 n+1}$. We have a map of homotopy-theoretic cofibrations

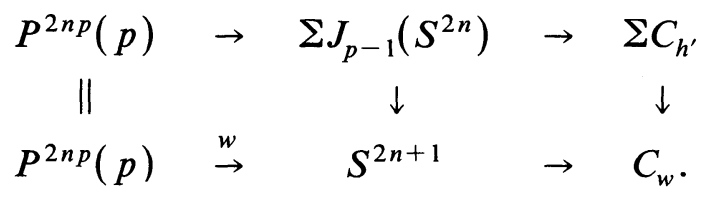

Diagram chasing shows that $P^{n}$ acts nontrivially in $C_{w}$ so that $\pi_{2 n(p-1)-2}^{s}$ has an element of Arf invariant 1.

$\mathrm{c} \Rightarrow \mathrm{b}^{\prime}$ : Let $w^{\prime}: P^{2 n(p-1)-1}\left(p^{r}\right) \rightarrow S^{0}$ be a stable map such that $P^{n}$ acts nontrivially in $C_{w^{\prime}}$. From the commutative square

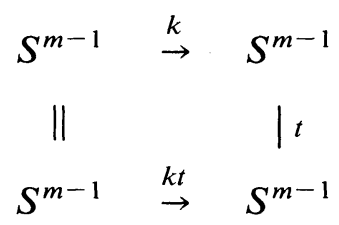

we get an induced map of homotopy-theoretic cofibres $P^{m}(k) \rightarrow P^{m}(k t)$. Let $w^{\prime \prime}$ be the composite

$$
P^{2 n(p-1)-1}(p) \rightarrow P^{2 n(p-1)-1}\left(p^{r}\right) \stackrel{w^{\prime}}{\rightarrow} S^{0} .
$$

We have a map of homotopy-theoretic cofibrations

$$
\begin{array}{ccccc}
P^{2 n(p-1)-1}(p) & \stackrel{w^{\prime \prime}}{\rightarrow} & S^{0} & \rightarrow & C_{w^{\prime \prime}} \\
\downarrow \alpha & & \| & & \downarrow \\
P^{2 n(p-1)-1}\left(p^{r}\right) & \stackrel{w^{\prime}}{\rightarrow} & S^{0} & \rightarrow & C_{w^{\prime}} .
\end{array}
$$

Since $\alpha$ induces an isomorphism on $H^{2 n(p-1)-1}$ diagram chasing in the long exact cohomology sequence shows that $P^{n}$ also acts nontrivially in $C_{w^{\prime \prime}}$. Since $\pi_{2 n p}\left(S^{2 n+1} ; Z / p Z\right)$ is stable, it contains a representative $w: P^{2 n p}(p) \rightarrow S^{2 n+1}$ for $w^{\prime \prime}$. Let $\tilde{w}: P^{2 n p-1}(p) \rightarrow \Omega S^{2 n+1}$ be the adjoint of $w$. For connectivity reasons, $\tilde{w}$ lifts to $v: P^{2 n p-1}(p) \rightarrow J_{p-1}\left(S^{2 n}\right)$. Let $F$ be the homotopy-theoretic fibre of $\gamma: J_{p-1}\left(S^{2 n}\right) \rightarrow C_{v}$. Let $\phi$ denote the mod $p$ Hurewicz homomorphism. We have a commutative diagram with exact rows 
where the bottom line is the Serre exact homology sequence. Diagram chasing in the cohomology Serre spectral sequences show that $H^{2 n p-2}\left(\Omega C_{v}\right) \rightarrow H^{2 n p-2}\left(\Omega J_{p-1}\left(S^{2 n}\right)\right)$ is the zero map, so its dual is also zero. According to the mod $p$ Hurewicz Isomorphism Theorem (see Neisendorfer [16], Theorem 3.8)

$\phi: \pi_{2 n p-3}(\Omega F ; Z / p Z) \rightarrow H_{2 n p-3}(\Omega F)$ is an isomorphism and $\phi: \pi_{2 n p-2}(\Omega F: Z / p Z) \rightarrow H_{2 n p-2}(\Omega F)$ is an epimorphism. So diagram chasing shows that $\phi: \pi_{2 n p-2}\left(\Omega J_{p-1}\left(S^{2 n}\right) ; Z / p Z\right) \rightarrow H_{2 n p-2}\left(\Omega J_{p-1}\left(S^{2 n}\right)\right)$ is an epimorphism. This statement is equivalent to $\left(b^{\prime}\right)$.

$\mathrm{b}^{\prime} \Rightarrow$ a: Let $h: P^{2 n p-2} \rightarrow \Omega J_{p-1}\left(S^{2 n}\right)$ induce a nonzero map on $H_{2 n p-2}$. Since $T_{*}$ is an isomorphism on $H_{2 n p-2}, T h \neq 0$. However the composite

$$
S^{2 n p-3} \stackrel{j}{\rightarrow} P^{2 n p-2}(p) \stackrel{h}{\rightarrow} \Omega J_{p-1}\left(S^{2 n}\right) \stackrel{T}{\rightarrow} \Omega S^{2 n p-1}
$$

must be null homotopic for connectivity reasons. So there exists $g: S^{2 n p-3} \rightarrow S^{2 n-1}$ such that

$$
\begin{array}{ccc}
S^{2 n p-3} & \stackrel{j}{\rightarrow} & P^{2 n p-2}(p) \\
\downarrow g & & \downarrow h \\
S^{2 n-1} & \stackrel{i}{\rightarrow} & \Omega J_{p-1}\left(S^{2 n}\right)
\end{array}
$$

is homotopy commutative. Since multiplication by $p$ kills $\operatorname{Im} j^{\# \text {, }}$ $i_{\#}(p g)=p i_{\#}(g)=p j^{\#}(h)=0$. Therefore $\Sigma^{2}(p g)=0$. But ker $\Sigma^{2}: \pi_{2 n p-3}\left(S^{2 n-1}\right) \rightarrow \pi_{2 n p-3}\left(\Omega^{2} S^{* n+1}\right)$ is $Z / p Z$ generated by $f$. So $p g=\lambda f$ for some $\lambda \in Z / p Z$. It remains to show that $\lambda \neq 0$. So suppose $\lambda=0$. Then there exists $\hat{g}: P^{2 n p-2}(p) \rightarrow S^{2 n-1}$ such that $g=\hat{g} j$. Since $(h-i \hat{g}) j=h j-i g=0$, there exists $e: S^{2 n p-2} \rightarrow \Omega J_{p-1}\left(S^{2 n}\right)$ such that $h-i g=e c$ where $c: P^{2 n p-2}(p) \rightarrow S^{2 n p-2}$ is the map which collapses $S^{2 n p-3}$ to a point. We have $T e c=T h-T i g=T h \neq 0$. Therefore $T e \neq 0$. But this implies that the fibration

$$
S^{2 n-1} \stackrel{i}{\rightarrow} \Omega J_{p-1}\left(S^{2 n}\right) \stackrel{T}{\rightarrow} \Omega S^{2 n p-1}
$$

has a cross-section, up to homotopy, and so $i$ induces a split monomorphism on homotopy groups. This is a contradiction since $f \neq 0$ but if $=0$. Therefore $\lambda=0$.

II. Self-maps. The purpose of this section is to prove the technical Theorem 2.3 which gives a sufficient condition for a map to be a homotopy equivalence. We begin with some algebraic preliminaries. 
LEMMA 2.1. Let $V$ be either a finite group or a finite dimensional vector space. Let $f: V \rightarrow V$. Let $W=\lim _{f} V$. Let $\theta: V \rightarrow W$ be the canonical map. Then $\theta$ is onto.

Proof. $\operatorname{Im} f^{n+1} \subset \operatorname{Im} f^{n}$ for all $n$. Since $V$ is finite or finite dimensional, these images stabilize. For convenience, write our direct system

$$
V_{0} \stackrel{f}{\rightarrow} V_{1} \stackrel{f}{\rightarrow} V_{2} \rightarrow \cdots \rightarrow V_{n} \rightarrow \cdots \rightarrow W
$$

where $V_{i}=V$ for all $i$. Let $w \in W$. Find a representative $x$ for $w$ in $V_{m}$ for some $m$. Pick $N$ large enough so that $\operatorname{Im} f^{N+k}=\operatorname{Im} f^{N}$ for all $k$. Now $f^{N} x$ belonging to $V_{N+m}$ is another representative of $w$. Since $f^{N} x \in \operatorname{Im} f^{N}$ $=\operatorname{Im} f^{N+m}, f^{N} x=f^{N+m} x^{\prime}$ for some $x^{\prime} \in V_{0}$, and $\theta\left(x^{\prime}\right)=w$.

Given an abelian group $G$, let $t(G)$ denote its torsion subgroup.

LEMMA 2.2. Let $G$ be a finitely generated $R$-module for some $R \subset Q$. Let $f: G \rightarrow G$. Let $H=\lim _{f} G$ and let $\theta: G \rightarrow H$ be the canonical map. Then

(1) coker $\theta$ is divisible.

(2) coker $\theta$ is a torsion group.

(3) $\left.t(H) \subset \operatorname{Im} \theta\right|_{t(G)}$.

(4) $G \stackrel{\theta}{\rightarrow} \operatorname{Im} \theta$ is a split epimorphism. Further, this splitting can be chosen to be natural when restricted to $t(\operatorname{Im} \theta)$.

REMARK. The second statement in (4) means the following:

Let

$$
\begin{array}{ccc}
G & \stackrel{f}{\rightarrow} & G \\
\downarrow \alpha & & \downarrow \alpha \\
G^{\prime} & \stackrel{f^{\prime}}{\rightarrow} & G^{\prime}
\end{array}
$$

be commutative. Then there exists $s: \operatorname{Im} \theta \rightarrow G$ and $s^{\prime}: \operatorname{Im} \theta^{\prime} \rightarrow G^{\prime}$ such that $\theta s=1, \theta^{\prime} s^{\prime}=1$, and

$$
\begin{array}{ccc}
t(\operatorname{Im} \theta) & \stackrel{s}{\rightarrow} & G \\
\downarrow & & \downarrow \alpha \\
t\left(\operatorname{Im} \theta^{\prime}\right) & \stackrel{s^{\prime}}{\rightarrow} & G^{\prime}
\end{array}
$$

commutes. 
Proof. Since

$$
G \stackrel{\theta}{\rightarrow} H \rightarrow \operatorname{coker} \theta \rightarrow 0
$$

is exact,

$$
G \otimes F \stackrel{\theta \otimes F}{\rightarrow} H \otimes F \rightarrow \operatorname{coker} \theta \otimes F \rightarrow 0
$$

is exact for all $F$. Thus for any field $F$, $\operatorname{coker} \theta \otimes F=0$ by Lemma 2.1.

Setting $F=Z / p Z$, we conclude that $p \operatorname{coker} \theta=\operatorname{coker} \theta$. Since this is true for all $p$, coker $\theta$ is divisible.

Setting $F=Q$, we get that $\operatorname{coker} \theta$ is a torsion group.

To show (3):

Let $H^{\prime}=\lim _{f} t(G)$ and let $H^{\prime \prime}=\lim _{\rightarrow} G / t(G)$. Since $\lim _{\rightarrow}$ preserves exactness we get

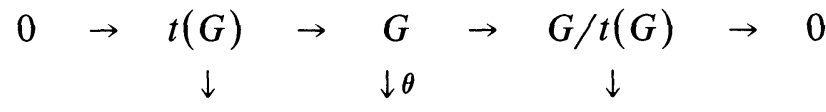

$$
\begin{aligned}
& 0 \rightarrow H^{\prime} \quad \rightarrow \quad H \quad \rightarrow \quad H^{\prime \prime} \quad \rightarrow \quad 0
\end{aligned}
$$

By Lemma 2.1, $t(G) \rightarrow H^{\prime}$ is onto, so it suffices to show that $t(H)$ goes to zero under $H \rightarrow H^{\prime \prime}$. But this is clear, since $H^{\prime \prime}$, being a direct limit of torsion-free groups is torsion-free.

To show (4):

Since $G$ is finitely generated, so is $\operatorname{Im} \theta$. Therefore

$$
\operatorname{Im} \theta \cong t(\operatorname{Im} \theta) \oplus F
$$

where $F$ is free. So any splitting defined on $t(\operatorname{Im} \theta)$ can be extended to $\operatorname{Im} \theta$. Thus it suffices to do the case where $G$ is a torsion group.

As in the proof of Lemma 2.1, write the direct system as

$$
G_{0} \stackrel{f}{\rightarrow} G_{1} \stackrel{f}{\rightarrow} G_{2} \rightarrow \cdots \rightarrow G_{n} \rightarrow \cdots \rightarrow H
$$

where $G_{i}=G$ for all $i$. Find $N$ such that $\operatorname{Im} f^{N}=\operatorname{Im} f^{N+k}$ for all $k$.

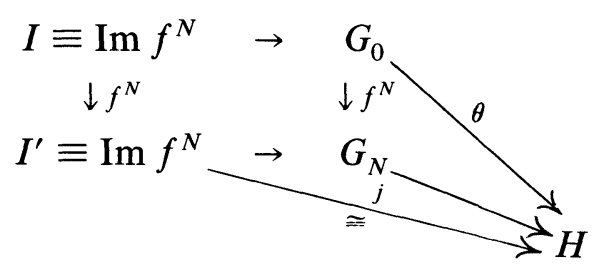

$H=\operatorname{Im} \theta$ since $G$ is a torsion group. Since $\operatorname{Im} f^{N}$ is stable, $j$ is an isomorphism. Also $f^{k}: \operatorname{Im} f^{N} \stackrel{\cong}{\operatorname{Im} f^{N+k}}$. In particular $\left.f^{k}\right|_{I}$ is injective 
for all $k$. So $\left.f^{N}\right|_{I}$ is injective. Since $I$ and $I^{\prime}$ are finite groups of the same order, $f^{N}: I \stackrel{\cong}{\rightarrow} I^{\prime}$. Thus $\theta$ splits.

Observe that our splitting depends upon our choice of $N$, but is canonical once $N$ has been chosen. Thus given a diagram

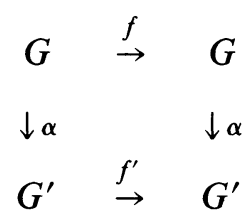

to get naturality, it is merely necessary to use the same $N$ in constructing the two splittings. Of course, $N$ must be chosen large enough so that both systems have stabilized.

Let $p$ be a prime. Let $X$ be a topological space. Let

$$
P_{*}^{n}: H_{q}(X ; Z / p Z) \rightarrow H_{q-2(p-1) n}(X ; Z / p Z)
$$

be the hom-dual of $P^{n}$ (respectively: $\mathrm{Sq}_{*}^{n}$ dual to $\mathrm{Sq}^{n}$ ). This defines a (left) $A_{*}$-module structure on $H_{*}(X ; Z / p Z)$ where $A_{*}$ is the opposite algebra of the $\bmod p$ Steenrod Algebra $A$. Let $\hat{A}$ denote the subalgebra of $A$ generated by $\left\{P^{n}\right\}_{n=1}^{\infty}$ (respectively: generated by $\left\{\mathrm{Sq}^{n}\right\}_{n=2}^{\infty}$ ) and let $\hat{A}_{*}$ denote its opposite algebra. Let $E^{r}(X)$ denote the $\bmod p$ homology Bockstein Spectral Sequence of $X$ and let $\beta^{(r)}$ be the $r$ th Bockstein. Let $\phi$ be the Hurewicz homomorphism and let

$$
r: \pi_{*}(X) \rightarrow \pi_{*}(X ; Z / p Z) \quad\left(\text { respectively: } H_{*}(X) \rightarrow H_{*}(X ; Z / p Z)\right)
$$

denote reduction $\bmod p$.

We now define some subspaces of $\mathrm{PH}_{*}(X ; Z / p Z)$, the primitives in the homology of $X$. Let

Ann $H_{*}(X ; Z / p Z)$

$$
=\left\{x \in P H_{*}(X ; Z / p Z) \mid x \in \operatorname{ker} P_{*}^{I} \text { for all } P_{*}^{I} \in A_{*}\right\} .
$$

Let

$\widehat{\operatorname{Ann}} H_{*}(X ; Z / p Z)$

$$
=\left\{x \in P H_{*}(X ; Z / p Z) \mid x \in \operatorname{ker} P_{*}^{I} \text { for all } P_{*}^{I} \in \hat{A}_{*}\right\} .
$$

Let

$S H_{*}(X ; Z / p Z)=\left\{x \in H_{*}(X ; Z / p Z) \mid x=f_{*}\left(\iota_{n}\right)\right.$ for some $\left.f: S^{n} \rightarrow X\right\}$. 
Let

$$
\begin{aligned}
& M H_{*}(X ; Z / p Z) \\
& =\left\{x \in \text { Ann } H_{*}(X ; Z / p Z) \mid \beta^{(r)} x=0 \text { for all } r\right. \text { and either } \\
& \text { (1) } x=0 \text {; or } \\
& \text { (2) } x \text { represents a nonzero class in } E^{\infty}(X) \text {; or } \\
& \text { (3) } x \text { represents a nonzero class in } E^{r}(X), \text { but } \\
& \\
& \left.x \in \operatorname{Im} \beta^{(r)}\left(\text { Ann } H_{*}(X ; Z / p Z)\right) \mid \text { for some } r\right\} .
\end{aligned}
$$

Let

$$
M S H_{*}(X ; Z / p Z)=M H_{*}(X ; Z / p Z) \cap S H_{*}(X ; Z / p Z) .
$$

The main result of this section is:

THEOREM 2.3. Let $X$ be a simply connected space having the homotopy type of a CW complex of finite type. Let $f: X \rightarrow X$. Suppose that $f_{*}^{N}$ restricted to $\operatorname{MSH}_{*}(X ; Z / p Z)$ is an injection for all $N$. Then $f_{(p)}$ is a homotopy equivalence.

Proof. Following Cohen, Moore, and Neisendorfer [9], §4, let $Y$ $=\lim _{f} X$, the infinite mapping telescope of $f$. Then $\pi_{*}(Y)=\lim _{f_{\sharp}} \pi_{*}(X)$ and $H_{*}(X)=\lim _{f_{*}} H_{*}(X)$. Similar statements hold for mod $p$ homotopy and homology.

We have a canonical map $\theta: X \rightarrow Y$ inducing the obvious maps on homotopy and homology. Let $F$ be the homotopy-theoretic fibre of $\theta: X \rightarrow Y$. Suppose $F_{(p)}$ is not contractible. Find $n$ such that $F_{(p)}$ is $(n-1)$ connected but not $n$ connected. We show that there exists a nonzero $x$ in $\operatorname{MSH}_{*}(X ; Z / p Z)$ such that $x \in \operatorname{Im} i_{*}$, where $i: F \rightarrow X$. Given such an $x$, the hypothesis implies that $\theta_{*} x \neq 0$. But this is impossible since $x \in \operatorname{Im} i_{*}$. Thus $F_{(p)}$ is contractible and so $\theta_{(p)}$ is a homotopy equivalence. Therefore $f_{(p)}$ is a homotopy equivalence. So it suffices to show the existence of such an $x$.

Case 1. $\pi_{n}(F ; Z / p Z) \neq 0$.

By the $\bmod p$ Hurewicz isomorphism, $H_{n}(F ; Z / p Z) \cong \pi_{n}(F ; Z / p Z)$ $\neq 0$. Let $x=i_{*}(w)$ for some nonzero $w$ in $H_{n}(F ; Z / p Z)$. By the Serre exact homology sequence and Lemma 2.1,

$$
i_{*}: H_{n}(F ; Z / p Z) \rightarrow H_{n}(X / Z / p Z)
$$


is injective so $x \neq 0$. Since

$$
w \in S H_{*}(F ; Z / p Z) \cap \text { Ann } H_{*}(F ; Z / p Z)
$$

and $\beta^{(s)} w=0$ for all $s, x$ has these properties also. It remains to show that $x$ satisfies either condition (2) or condition (3) in the definition of $M H_{*}(X ; Z / p Z)$.

If $x$ persists to a nonzero element in $E^{\infty}(X)$ we are finished, so suppose not. Then for some $m, x$ is nonzero in $E^{m}(X)$, but $x \in \operatorname{Im} \beta^{(m)}$. We must show that $x \in \beta^{(m)}\left(\widehat{\operatorname{Ann}} H_{*}(X ; Z / p Z)\right)$.

Find $b \in H_{n}(X)$ such that order $b=p^{m}$ and $r b=x$. We adjust $b$ so that it lies in $\operatorname{Im} i_{*}$ as follows:

By Lemma 2.2, find splittings such that

$$
\begin{array}{ccccc}
H_{n}(Y) & \supset & t\left(\operatorname{Im} \theta_{*}\right) & \stackrel{s}{\rightarrow} & H_{n}(X) \\
& & \downarrow r & & \downarrow r \\
H_{n}(Y ; Z / p Z) & \supset & \operatorname{Im} \theta_{*} & \stackrel{s}{\rightarrow} & H_{n}(X ; Z / p Z)
\end{array}
$$

commutes. Since $b \in t\left(H_{n}(X)\right), \theta_{*} b \in t(\operatorname{Im} \theta)$ so $s \theta_{*} b$ is defined. Let $b^{\prime}=b-s \theta_{*} b$. We have

$$
r b^{\prime}=r b-r s \theta_{*} b=x-s r \theta_{*} b=x-s \theta_{*} r b=x-s \theta_{*} x=x
$$

since $\theta_{*} x=\theta_{*} i_{*} w=0$. Because $p^{m} b=0$, it follows that $p^{m} b^{\prime}=0$. Since $r b^{\prime}=x$ and $x$ is nonzero in $E^{m}(X)$, order $b^{\prime}=p^{m}$. Finally,

$$
\boldsymbol{\theta}_{*} b^{\prime}=\boldsymbol{\theta}_{*} b-\boldsymbol{\theta}_{*} s \boldsymbol{\theta}_{*} b=\boldsymbol{\theta}_{*} b-\boldsymbol{\theta}_{*} b=0
$$

so $b^{\prime}=i_{*} a$ for some $a \in H_{n}(F)$ by the Serre exact homology sequence

$$
\cdots \rightarrow H_{n+1}(X) \stackrel{\theta_{*}}{\rightarrow} H_{n+1}(Y) \stackrel{\partial}{\rightarrow} H_{n}(F) \stackrel{i}{\rightarrow} H_{n}(X) \stackrel{\theta_{*}}{\rightarrow} H_{n}(Y) \rightarrow \cdots .
$$

Next we adjust $a$ so that order $a=\operatorname{order} b^{\prime}$.

Since $i_{*}\left(p^{m} a\right)=p^{m} b^{\prime}=0, p^{m} a=\partial y$ for some $y$ belonging to $\operatorname{coker} \theta_{*}: H_{n+1}(X) \rightarrow H_{n+1}(Y)$. By Lemma 2.2, $\operatorname{coker} \theta_{*}$ is divisible so $y=p^{m} y^{\prime}$ for some $y^{\prime}$ belonging to $\operatorname{coker} \theta_{*}$. Let $a^{\prime}=a-\partial y^{\prime}$. Then $i_{*} a^{\prime}=i_{*} a-i_{*} \partial y^{\prime}=b^{\prime}$ and $p^{m} a^{\prime}=p^{m} a-p^{m} \partial y^{\prime}=p^{m} a-\partial y=0$.

Let $g$ be the image of $a^{\prime}$ under

$$
H_{n}(F) \rightarrow H_{n}\left(F_{(p)}\right) \stackrel{\theta^{-1}}{\cong} \pi_{n}\left(F_{(p)}\right) \text {. }
$$

Since $p^{m} g=0, g$ extends to $\hat{g}: P^{n+1}\left(p^{m}\right) \rightarrow F_{(p)}$. 
Let $u_{n}$ and $v_{n+1}$ be a basis for $\tilde{H}_{*}\left(P^{n+1}\left(p^{m}\right) ; Z / p Z\right)$ such that $\beta^{(m)} v=u$. Then under the composite

$$
\begin{aligned}
& H_{*}\left(P^{n+1}\left(p^{m}\right) ; Z / p Z\right) \stackrel{g_{*}}{\rightarrow} H_{*}\left(F_{(p)} ; Z / p Z\right) \stackrel{i_{*}}{\rightarrow} H_{*}\left(X_{(p)} ; Z / p Z\right) \\
& \cong H_{*}(X ; Z / p Z)
\end{aligned}
$$

$u$ goes to $x$. So if we let $x^{\prime}$ be the image of $v$, then

$$
x^{\prime} \in \widehat{\operatorname{Ann}} H_{*}(X ; Z / p Z) \text { and } \beta^{(m)} x^{\prime}=x .
$$

Case 2. $\pi_{n}(F ; Z / p Z)=0$.

According to the Universal Coefficient Theorem, (see Neisendorfer [16], Proposition 1.4), there is a short exact sequence

$$
0 \rightarrow \pi_{n}\left(F_{(p)}\right) \otimes Z / p Z \rightarrow \pi_{n}\left(F_{(p)} ; Z / p Z\right) \rightarrow \operatorname{Tor}\left(\pi_{n-1}\left(F_{(p)}\right) ; Z / p Z\right) \rightarrow 0 .
$$

So

$$
\pi_{n}\left(F_{(p)}\right) \otimes Z / p Z \cong \pi_{n}\left(F_{(p)} ; Z / p Z\right) \cong \pi_{n}(F ; Z / p Z)=0
$$

Therefore $\pi_{n}\left(F_{(p)}\right)$ is divisible. Since $\pi_{n}\left(X_{(p)}\right)$ is a finitely generated $Z_{(p)}$-module, it follows that $i_{\#}: \pi_{n}\left(F_{(p)}\right) \rightarrow \pi_{n}\left(X_{(p)}\right)$ is the zero map. Thus

$$
\pi_{n}\left(F_{(p)}\right) \cong \operatorname{coker} \theta_{\#}: \pi_{n+1}\left(X_{(p)}\right) \rightarrow \pi_{n+1}\left(Y_{(p)}\right) .
$$

Since $F_{(p)}$ is $(n-1)$ connected but not $n$ connected we can find a nonzero $a$ in $\pi_{n}\left(F_{(p)}\right)$. By Lemma $2.2, \pi_{n}\left(F_{(p)}\right)$ is a torsion group so $p^{s} a=0$ for some $s$. By replacing $a$ by $p^{s-1} a$, we may assume that $p a=0$.

Because $i_{\#}(a)=0, a=\partial y$ for some $y$ belonging to $\pi_{n+1}\left(Y_{(p)}\right)$. Since $\partial(p y)=p a=0, p y=\theta_{\#}(g)$ for some $g$ in $\pi_{n+1}\left(X_{(p)}\right)$. Let $x$ be the image of $g$ under

$$
\begin{aligned}
\pi_{n+1}\left(X_{(p)}\right) & \stackrel{r}{\rightarrow} \pi_{n+1}\left(X_{(p)} ; Z / p Z\right) \stackrel{\phi}{\rightarrow} H_{n+1}\left(X_{(p)} ; Z / p Z\right) \\
& \cong H_{n+1}(X ; Z / p Z) .
\end{aligned}
$$

By construction $x \in S H_{*}(X ; Z / p Z)$. We next show that $x \in \operatorname{Im} i_{*}$ and $x \neq 0$.

Let $S^{n}\{p\}$ denote the homotopy-theoretic fibre of $p: S^{n} \rightarrow S^{n}$. Let $k$ : $P^{n}(p) \rightarrow S^{n}\{p\}$ be the inclusion of the $n$-skeleton into $S^{n}\{p\}$. Let $g^{\prime}$ and 
$y^{\prime}$ be the adjoints of $g$ and $y$ respectively. From the homotopy commutative square

$$
\begin{array}{ccc}
S^{n} & \stackrel{g^{\prime}}{\rightarrow} & \Omega X_{(p)} \\
\downarrow p & & \downarrow \Omega \theta \\
S^{n} & \stackrel{y^{\prime}}{\rightarrow} & \Omega Y_{(p)}
\end{array}
$$

we get an induced map of homotopy-theoretic fibres $b: S^{n}\{p\} \rightarrow \Omega F_{(p)}$.

We have a homotopy commutative diagram

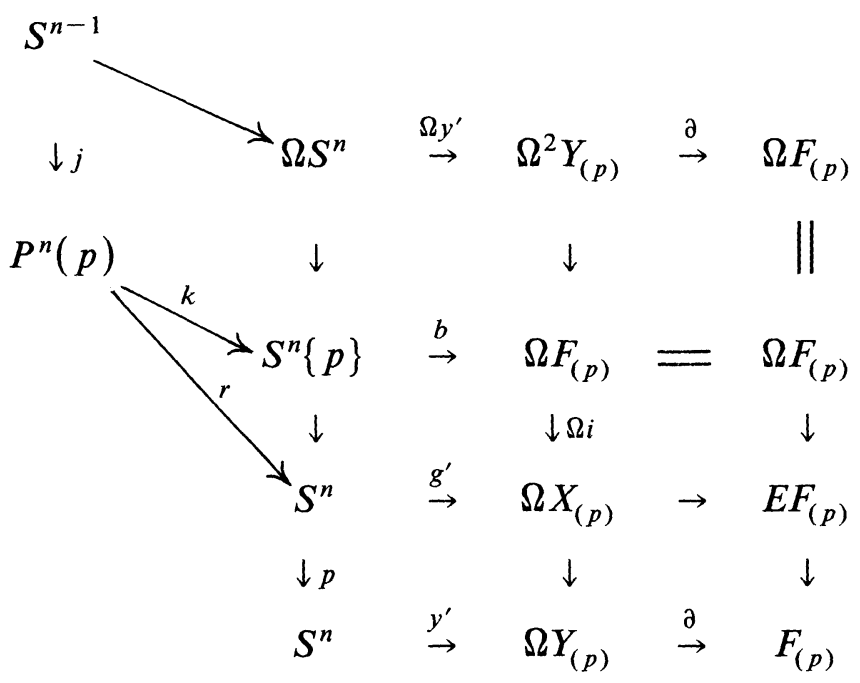

where the columns are homotopy-theoretic fibrations and the collapse map, denoted $r$ here, induces reduction $\bmod p$ on homotopy groups.

$$
\begin{array}{ccccc}
\pi_{n+1}\left(F_{(p)} ; Z / p Z\right) & \stackrel{i_{\#}}{\rightarrow} & \pi_{n+1}\left(X_{(p)} ; Z / p Z\right) & \cong & \pi_{n+1}(X ; Z / p Z) \\
\downarrow \phi & & \downarrow_{\phi} & & \downarrow \phi \\
H_{n+1}\left(F_{(p)} ; Z / p Z\right) & \stackrel{i_{*}}{\rightarrow} & H_{n+1}\left(X_{(p)} ; Z / p Z\right) & \cong & H_{n+1}(X ; Z / p Z)
\end{array}
$$

From Lemma 2.1, the long exact homotopy sequence, and the Serre exact homology sequence, $i_{\#}$ and $i_{*}$ are injective. Also, since we are doing Case 2, the leftmost map is an isomorphism by the $\bmod p$ Hurewicz Isomorphism Theorem. 
From (1) we see that $r^{\#}\left(g^{\prime}\right)=(\Omega i)_{\#}(b k)$. In other words, in $\pi_{n+1}\left(X_{(p)} ; Z / p Z\right), r g=i_{\#}($ adjoint of $b k)$. Therefore $x \in \operatorname{Im} i_{*}$ and to show $x \neq 0$, by diagram chasing from (2), it suffices to show $b k \neq 0$. But following (1) across the top, $b k j$ is the adjoint of $a$, which is nonzero. Thus $x \neq 0$.

To show $x \in M H_{*}(X ; Z / p Z)$ it now suffices to show that $x$ represents a nonzero element in $E^{\infty}(X)$. Suppose this is not true. Then there exists $z$ belonging to $H_{n+1}\left(X_{(p)}\right)$ such that $r z=x$ and order $z<\infty$. Let $s$ be the splitting of Lemma 2.2 chosen so that

$$
\begin{array}{ccccc}
H_{n+1}\left(Y_{(p)}\right) & \supset & t\left(\operatorname{Im} \theta_{*}\right) & \stackrel{s}{\rightarrow} & H_{n+1}\left(X_{(p)}\right) \\
\downarrow r & & \downarrow r & & \downarrow r \\
H_{n+1}\left(Y_{(p)} ; Z / p Z\right) & \supset & \operatorname{Im} \theta_{*} & \stackrel{s}{\rightarrow} & H_{n+1}\left(X_{(p)} ; Z / p Z\right)
\end{array}
$$

commutes. Since $z \in t\left(H_{n+1}\left(X_{(p)}\right)\right), s \theta_{*} z$ is defined. Let $z^{\prime}=z-s \theta_{*} z$. Since $x \in \operatorname{Im} i_{*}, \theta_{*} x=0$ and so

$$
r z^{\prime}=r z-r s \theta_{*} z=x-s \theta_{*} x=x .
$$

Also

$$
\theta_{*} z^{\prime}=\theta_{*} z-\theta_{*} s \theta_{*} z=\theta_{*} z-\theta_{*} z=0
$$

so $z^{\prime}=i_{*}(w)$ for some $w \in H_{n+1}\left(F_{(p)}\right)$.

We have $i_{*} r w=r z^{\prime}=x=i_{*} \phi(b k)$. Since $i_{*}$ is a monomorphism, this implies $r w=\phi(b k)$. Therefore diagram chasing from

$$
\begin{aligned}
& \ldots \quad \rightarrow \quad \pi_{n+1}\left(F_{(p)}\right) \quad \stackrel{r}{\rightarrow} \quad \pi_{n+1}\left(F_{(p)} ; Z / p Z\right) \stackrel{j^{\#}}{\rightarrow} \pi_{n}\left(F_{(p)}\right) \quad \rightarrow \quad \ldots \\
& \downarrow \phi \quad \cong \downarrow \phi \quad \cong \downarrow \phi \\
& \cdots \quad \rightarrow \quad H_{n+1}\left(F_{(p)}\right) \stackrel{r}{\rightarrow} \quad H_{n+1}\left(F_{(p)} ; Z / p Z\right) \quad \rightarrow \quad H_{n}\left(F_{(p)}\right) \quad \rightarrow \quad \cdots
\end{aligned}
$$

shows that $j^{\#}(b k)=0$. But as noted earlier, $j^{\#}(b k)$ is the adjoint of $a$, which is nonzero. This is a contradiction, so $x$ must be nonzero in $E^{\infty}(X)$.

III. Some atomic spaces. In this section homology is assumed to be with $Z / p Z$ coefficients unless stated otherwise.

Definition 3.1. Let $X_{(p)}$ be $(n-1)$ connected. Then $X$ is called $\bmod p$ atomic if:

(1) $H_{n}(X)=Z / p Z$

(2) $f: X \rightarrow X$ such that $f$ induces an isomorphism on $H_{n}(X)$ implies that $f_{(p)}$ is a homotopy equivalence. 
$X$ is called atomic if it is $\bmod p$ atomic for all $p$.

Clearly $X$ atomic implies that $X$ is indecomposable in the sense that if $X \approx Y \times Z$ then either $Y \approx\{$ pt. $\}$ or $Z \approx\{$ pt. $\}$.

Some trivial examples of atomic spaces are $S^{n}$ and $\Omega S^{2 n+1}$. A nontrivial example is given by:

Theorem 3.2 (Cohen and Mahowald). $\Omega^{2} S^{2 n+1}$ is atomic for $n>1$.

Proof. See [5].

REMARK 1. The corresponding statement for $n=1$ is that $\Omega^{2} S^{3}\langle 3\rangle$ is atomic. This is also proved in [5].

REMARK 2. If $p>2, \Omega^{2} S^{2 n}$ cannot be $\bmod p$ atomic since after localization at $p$,

$$
\Omega S^{2 n} \approx S^{2 n-1} \times \Omega S^{4 n-1} .
$$

From now on we shall assume that $p$ is odd and we shall take the term "atomic" to mean $\bmod p$ atomic for all odd primes $p$.

THEOREM 3.3. Assume $n>1$. Then $\operatorname{MSH}_{q}\left(\Omega S^{2 n+1}\{p\}\right)=0$ for $q>$ $2 n-1$ if and only if there are no elements of Arf invariant $1 \bmod p$ in $\pi_{2 n(p-1)-2}^{s}$.

COROLlaRY 3.4. $\Omega S^{2 n+1}\{p\}$ is atomic for all $n$ such that $\pi_{2 n(p-1)-2}^{s}$ has no elements of Arf invariant $1 \mathrm{mod} p$. In particular if $n \neq p^{k}$ for some $k$ then $\Omega S^{2 n+1}\{p\}$ is atomic.

Applying the theorem of Ravenel ([19]) gives:

COROLlaRY 3.5. If $p \geq 5$ and $n \neq 1$ or $p$ then $\Omega S^{2 n+1}\{p\}$ is atomic.

Proof of Theorem 3.3. As a Hopf Algebra over the Steenrod Algebra, $H_{*}\left(\Omega S^{2 n+1}\{p\}\right)$ is given by the following (see [6]):

$$
H_{*}\left(\Omega S^{2 n+1}\{p\}\right)=\bigotimes_{k=0}^{\infty} E\left[a_{2 n p^{k}-1}\right] \otimes \bigotimes_{k=1}^{\infty} Z / p Z\left[b_{2 n p^{k}-2}\right] \otimes Z / p Z\left[c_{2 n}\right]
$$


with the generators primitive and

$$
\begin{array}{rlrl}
\beta c_{2 n} & =a_{2 n-1}, & & \\
\beta a_{2 n p^{k-1}} & =b_{2 n p^{k}-2}, & & k \geq 1, \\
P_{*}^{1} a_{2 n p^{k}-1} & =P_{*}^{1} b_{2 n p-2}=P_{*}^{1} c_{2 n}=0, & & \\
P_{*}^{1} b_{2 n p^{k-2}} & =-\left(b_{2 n p^{k-1}-2}\right)^{p}, & & k \geq 2, \\
P_{*}^{p^{r}} & \equiv 0, & r \geq 1 .
\end{array}
$$

From this description we see that $a_{2 n-1}$ and $b_{2 n p-2}$ form a basis for $M H_{*}\left(\Omega S^{2 n+1}\{p\}\right)$. It remains to show that $b_{2 n p-2} \in S H_{*}\left(\Omega S^{2 n+1}\{p\}\right)$ if and only if there exist elements of Arf invariant $1 \bmod p$ in $\pi_{2 n(p-1)-2}^{s}$.

Suppose first that there exists $h: S^{2 n p-2} \rightarrow \Omega S^{2 n+1}\{p\}$ such that $h_{*}\left(\iota_{2 n p-2}\right)=b_{2 n p-2}$. Let $h^{\prime}: S^{2 n p-3} \rightarrow \Omega^{2} S^{2 n+1}\{p\}$ be the adjoint of $h$. Let $\sigma_{*}$ denote the homology suspension. Then $\sigma_{*} h_{*}^{\prime}\left(\iota_{2 n p-3}\right)=h_{*}\left(\iota_{2 n p-2}\right)$ $\neq 0$, so $h_{*}^{\prime}\left(\iota_{2 n p-3}\right) \neq 0$.

Localize at $p$ and let $\pi: \Omega^{2} S^{2 n+1} \rightarrow S^{2 n-1}$ be the map constructed by Cohen, Moore, and Neisendorfer in [7]. According to Cohen, Moore, and Neisendorfer ([8], Theorem 1.1) $\pi \circ \Sigma^{2}=p: S^{2 n-1} \rightarrow S^{2 n-1}$ and $\Sigma^{2} \circ \pi=$ $p: \Omega^{2} S^{2 n+1} \rightarrow \Omega^{2} S^{2 n+1}$. Thus we get a map of homotopy-theoretic fibrations

$$
\begin{array}{ccccccc}
\Omega^{3} S^{2 n+1} & \rightarrow & \Omega^{2} S^{2 n+1}\{p\} & \stackrel{k}{\rightarrow} & \Omega^{2} S^{2 n+1} & \stackrel{p}{\rightarrow} & \Omega^{2} S^{2 n+1} \\
\| & & \downarrow i & & \downarrow \pi & & \| \\
\Omega^{3} S^{2 n+1} & \rightarrow & C(n) & \stackrel{j}{\rightarrow} & S^{2 n-1} & \stackrel{\Sigma^{2}}{\rightarrow} & \Omega^{2} S^{2 n+1} .
\end{array}
$$

From the far left square we can see that $i_{*}$ induces an isomorphism on $H_{2 n p-3}$ so $i_{*} h_{*}^{\prime} \neq 0$ on $H_{2 n p-3}$. Therefore by the Hurewicz isomorphism, $i h^{\prime}$ is a generator of $\pi_{2 n p-3}(C(n))$. Let $f=j i h_{*}^{\prime}$. Since $\Sigma^{2}: \pi_{2 n p-3}\left(S^{2 n-1}\right) \rightarrow \pi_{2 n p-3}\left(\Omega^{2} S^{2 n+1}\right)$ is onto, $k h^{\prime}=\Sigma^{2} g$ for some $g$ belonging to $\pi_{2 n p-3}\left(S^{2 n-1}\right)$. Thus $f^{\prime}=j i h^{\prime}=\pi k h^{\prime}=\pi \Sigma^{2} g=p g$. So there exist elements of Arf invariant $1 \bmod p$ in $\pi_{2 n(p-1)-2}^{s}$ by Theorem 1.1.

Conversely if there exist elements of Arf invariant $1 \bmod p$ in $\pi_{2 n(p-1)-2}^{s}$ then there exists $g$ in $\pi_{2 n p-3}\left(S^{2 n-1}\right)$ such that $p g=j s$ where $s$ is a generator of $\pi_{2 n p-3}(C(n))$. The middle square of $(*)$ is a homotopy pullback and $j s=p g=\pi \Sigma^{2} g$, so $s$ and $\Sigma^{2} g$ can be used to define 
a map $h^{\prime}: S^{2 n p-3} \rightarrow \Omega^{2} S^{2 n+1}\{p\}$. It is easy to see that if we let $h: S^{2 n p-2} \rightarrow \Omega S^{2 n+1}\{p\}$ be the adjoint of $h^{\prime}$ then $h_{*}\left(\iota_{2 n p-2}\right)=b_{2 n p-2}$ so that $b_{2 n p-2} \in S H_{*}\left(\Omega S^{2 n+1}\{p\}\right)$.

Assuming $n>1$, as a Hopf algebra

$$
\begin{aligned}
H_{*}\left(\Omega^{3} S^{2 n+1}\right) \cong & \bigotimes_{\substack{k \geq 1 \\
j \geq 0}} E\left[a_{2\left(n p^{k}-1\right) p^{j-1}}\right] \\
& \otimes \bigotimes_{\substack{k \geq 1 \\
j \geq 1}} Z / p Z\left[b_{2\left(n p^{k}-1\right) p^{j-2}}\right] \otimes \bigotimes_{k \geq 0} Z / p Z\left[c_{2 n p^{k}-2}\right]
\end{aligned}
$$

with the generators primitive. (See [6]). For convenience we will write these generators as $a_{n}(j, k), b_{n}(j, k)$, and $c_{n}(k)$ respectively and when no confusion is possible, we will drop the $n$.

From the Nishida relations the actions of $\beta$ and $P_{*}^{1}$ are as follows:

$$
\begin{aligned}
\beta a(j, k) & =b(j, k), & & j \geq 1, \\
\beta c(k) & =a(0, k), & & k \geq 1, \\
\beta a(0, k) & =\beta b(j, k)=\beta c(0)=0, & & \\
P_{*}^{1} b(j, k) & =(b(j-1), k)^{p}, & & j \geq 2, \\
P_{*}^{1} c(k) & =-(c(k-1))^{p}, & & k \geq 2, \\
P_{*}^{1} c(1) & =(n-1)(c(0))^{p}, & & \\
P_{*}^{1} a(j, k) & =P_{*}^{1} b(1, k)=P_{*}^{1} c(0)=0 . & &
\end{aligned}
$$

Since $H_{*}\left(\Omega^{3} S^{2 n+1}\right) \rightarrow H_{*}(C(n))$ simply projects off of $c(0)$, we can deduce the action of $\beta$ and $P_{*}^{1}$ in $H_{*}(C(n))$. We easily calculate that $M H_{*}\left(\Omega^{3} S^{2 n+1}\right)$ must be contained in the subspace generated by the elements
(1) $a(0,1)$,
(2) $b(1, k), k \geq 1$,
(3) $(c(0))^{p^{t}}, t \geq 0$.

Similarly $M H_{*}(C(n))$ is contained in the subspace generated by

(1) $a(0,1)$,

(2) $b(1, k), k \geq 1$.

Lemma 3.6. Assume $n>1$. Then for all $k, b(k, 1)$ does not belong to $S H_{*}\left(\Omega^{3} S^{2 n+1}\right)$ and its image does not belong to $\mathrm{SH}_{*}(C(n))$. 
Proof. If $b(1, k)$ were spherical in $S H_{*}\left(\Omega^{3} S^{2 n+1}\right)$ then its image in $H_{*}(C(n))$ would be spherical also, so it suffices to prove the second statement.

Toda ([22]) constructs a homotopy-theoretic fibration

$$
\Omega S^{2 n p-1}\{p\} \rightarrow C(n) \stackrel{H}{\rightarrow} C(n p) .
$$

(See also [20], Theorem 13.) Since $H_{*}\left(b_{n}(1, k)\right)=b_{n p}(1, k-1)$ for $k \geq 2$, if $b_{n}(1, k) \in S H_{*}(C(n))$ then $b_{n p}(1, k-1) \in S H_{*}(C(n p))$. So it suffices to prove the lemma for $k=1$.

Suppose that $b(1,1) \in S H_{*}(C(n))$. Because $|b(1,1)|<2 n p^{2}-4$ which is the connectivity of $C(n p), b(1,1)$ lifts to an element in $S H_{2 m p-2}\left(\Omega S^{2 m+1}\{p\}\right)$ for $m=n p-1$. But this contradicts Theorem 3.3, since $m$ is not a power of $p$. Therefore $b(1,1) \notin S H_{*}(C(n))$.

THEOREM 3.7. $C(n)$ is atomic for $n>1$.

Proof. Using Lemma 3.6 we see that $\operatorname{MSH}_{q}(C(n))=0$ for $q>$ $2 n p-3$ so this is immediate from Theorem 2.3.

THEOREM 3.8. $\Omega^{3} S^{2 n+1}$ is atomic for $n>1$.

Proof. Let $f: \Omega^{3} S^{2 n+1} \rightarrow \Omega^{3} S^{2 n+1}$ such that $f$ induces an isomorphism on $H_{2 n-2}\left(\Omega^{3} S^{2 n+1}\right)$. It is well known that $a(0,1) \notin S H_{*}\left(\Omega^{3} S^{2 n+1}\right)$ by the non-existence of elements of Hopf invariant 1 in $\pi_{2 n(p-1)-1}$, the argument being similar to the proof of the $b^{\prime} \Rightarrow c$ step in the proof of Theorem 1.1. Thus using Lemma 3.6, $\mathrm{MSH}_{*}\left(\Omega^{3} S^{2 n+1}\right)$ is contained in the subspace generated by $\left\{\left(c(0)^{p^{t}}\right)\right\}_{t=0}^{\infty}$. So by Theorem 2.3 , to show that $f$ is a homotopy equivalence it suffices to show that $f_{*}^{N}\left((c(0))^{p^{t}}\right) \neq 0$ for all $t$ and for all $N$. But this is easy to see by considering the action of $f^{*}$ on cohomology with $Z_{(p)}$ coefficients.

Remark. F. Cohen, F. Peterson, and the author have recently shown that $\Omega^{3} S^{2 n+1}$ is also mod 2 atomic for $n>1$, using different techniques.

For our final application, suppose $n>1$ and let $D(n)$ denote the homotopy-theoretic fibre of $\pi: \Omega^{2} S^{2 n+1} \rightarrow S^{2 n-1}$. Using [8], Lemma 2.1 and the fact that

$$
\Sigma^{2} \circ \pi=p: \Omega^{2} S^{2 n+1} \rightarrow \Omega^{2} S^{2 n+1}
$$


we can construct a homotopy-theoretic fibration

$$
D(n) \rightarrow \Omega^{2} S^{2 n+1}\{p\} \rightarrow C(n) .
$$

The main theorem of [20] asserts that this fibration splits when $n=p$.

THEOREM 3.9. The homotopy-theoretic fibration

$$
D(n) \rightarrow \Omega^{2} S^{2 n+1}\{p\} \rightarrow C(n)
$$

cannot split unless $\pi_{2 n(p-1)-2}^{s}$ contains an element of Arf invariant 1 .

Proof. If the fibration splits then the generator of $\pi_{2 n p-3}(C(n))$ lifts to a map from $S^{2 n p-3}$ to $\Omega^{2} S^{2 n+1}\{p\}$ and this results in the same situation as occurred in the proof of Theorem 3.3

\section{REFERENCES}

[1] J. F. Adams, On the non-existence of elements of Hopf invariant one, Ann. of Math., 72 (1960), 20-104.

[2] J. Aguadé, Cohomology algebras with two generators, (to appear).

[3] A. K. Bousfield, and D. M. Kan, Homotopy Limits, Completions and Localizations, Lecture Notes in Math., 304, springer-Verlag (1972).

[4] W. Browder, Manifolds of Kervaire invariant one, Ann. of Math., 90 (1969), 157-186.

[5] F. R. Cohen, and M. E. Mahowald, $A$ remark on the self-maps of $\Omega^{2} S^{2 n+1}$, Indiana Univ. Math. J., 30 (1981), 583-588.

[6] F. R. Cohen, T. J. Lada, and J. P. May, The Homology of Iterated Loops Spaces, Lecture Notes in Math., 533 Springer-Verlag (1976).

[7] F. R. Cohen, J. C. Moore, and J. A. Neisendorfer, Torsion in homotopy groups, Ann. of Math., 109 (1979), 121-168.

[8] The double suspension and exponents of the homotopy groups of spheres, Ann. of Math., 110 (1979), 549-565.

[9] __ Moore spaces have exponents, (to appear).

[10] D. Husemoller, Fibre Bundles, second edition, Graduate Texts in Math., 20 Springer-Verlag (1975).

[11] I. James, Reduced product spaces, Ann. of Math., 62 (1955), 170-197.

[12] _ On the suspension triad, Ann. of Math., 63 (1956), 191-247.

[13] _ The suspension triad of a sphere, Ann. of Math., 63 (1956), 407-429.

[14] A. Liulevicius, The factorization of cyclic reduced powers by secondary cohomology operations, Mem. Amer. Math. Soc., 42 (1962).

[15] M. E. Mahowald, Some remarks on the Kervaire invariant problem from the homotopy point of view, Proceedings of Symposia in Pure Mathematics XXII, Madison, Wisconsin, 1970, 165-169.

[16] J. A. Neisendorfer, Primary homotopy theory, Mem. Amer. Math. Soc., 232 (1980).

[17] , 3-primary exponents, Proc. Camb. Phil. Soc., 90 (1981), 63-83.

[18] F. Peterson, Thesis, Princeton University (1955).

[19] D. C. Ravenel, The non-existence of odd primary Arf invariant elements in stable homotopy, Math. Proc. Camb. Phil. Soc., 83 (1978), 429-443. 
[20] P. S. Selick, $A$ decomposition of $\pi_{*}\left(S^{2 p+1} ; Z / p Z\right)$, Topology, 20 (1981), 175-177.

[21] _ A spectral sequence concerning the double suspension, Invent. Math., 64 (1981), 15-24.

[22] N. Shimada, and T. Yamanoshita, On the triviality of the mod-p Hopf invariant, Japan J. Math., 31 (1961), 1-25.

[23] H. Toda, On the double suspension $E^{2}$, J. Institute Polytech. Osaka City Univ., Ser. A, 7 (1956), 103-145.

Received July 24, 1981.

UNIVERSITY OF WESTERN ONTARIO

LONDON, CANADA N6A 5B9 


\title{
PACIFIC JOURNAL OF MATHEMATICS EDITORS
}

\author{
Donald BabBitT (Managing Editor) \\ University of California \\ Los Angeles, CA 90024 \\ Hugo Rossi \\ University of Utah \\ Salt Lake City, UT 84112 \\ C. C. Moore and Arthur Ogus \\ University of California \\ Berkeley, CA 94720
}

\author{
J. DugundiI \\ Department of Mathematics \\ University of Southern California \\ Los Angeles, CA 90089-1113 \\ R. FINN and H. SAMELSON \\ Stanford University \\ Stanford, CA 94305
}

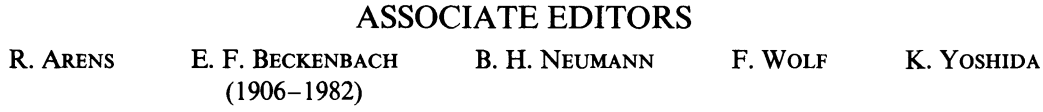

\section{SUPPORTING INSTITUTIONS}

UNIVERSITY OF ARIZONA
UNIVERSITY OF BRITISH COLUMBIA
CALIFORNIA INSTITUTE OF TECHNOLOGY
UNIVERSITY OF CALIFORNIA
MONTANA STATE UNIVERSITY
UNIVERSITY OF NEVADA, RENO
NEW MEXICO STATE UNIVERSITY
OREGON STATE UNIVERSITY

\author{
UNIVERSITY OF OREGON \\ UNIVERSITY OF SOUTHERN CALIFORNIA \\ STANFORD UNIVERSITY \\ UNIVERSITY OF HAWAII \\ UNIVERSITY OF TOKYO \\ UNIVERSITY OF UTAH \\ WASHINGTON STATE UNIVERSITY \\ UNIVERSITY OF WASHINGTON
}

The Supporting Institutions listed above contribute to the cost of publication of this Journal, but they are not owners or publishers and have no responsibility for its content or policies.

Mathematical papers intended for publication in the Pacific Journal of Mathematics should be in typed form or offset-reproduced (not dittoed), double spaced with large margins. Please do not use built up fractions in the text of the manuscript. However, you may use them in the displayed equations. Underline Greek letters in red, German in green, and script in blue. The first paragraph must be capable of being used separately as a synopsis of the entire paper. In particular it should contain no bibliographic references. Please propose a heading for the odd numbered pages of less than 35 characters. Manuscripts, in triplicate, may be sent to any one of the editors. Please classify according to the scheme of Math. Reviews, Index to Vol. 39. Supply name and address of author to whom proofs should be sent. All other communications should be addressed to the managing editor, or Elaine Barth, University of California, Los Angeles, California 90024.

There are page-charges associated with articles appearing in the Pacific Journal of Mathematics. These charges are expected to be paid by the author's University, Government Agency or Company. If the author or authors do not have access to such Institutional support these charges are waived. Single authors will receive 50 free reprints; joint authors will receive a total of 100 free reprints. Additional copies may be obtained at cost in multiples of 50 .

The Pacific Journal of Mathematics is issued monthly as of January 1966. Regular subscription rate: $\$ 132.00$ a year (6 Vol., 12 issues). Special rate: $\$ 66.00$ a year to individual members of supporting institutions.

Subscriptions, orders for numbers issued in the last three calendar years, and changes of address should be sent to Pacific Journal of Mathematics, P.O. Box 969, Carmel Valley, CA 93924, U.S.A. Old back numbers obtainable from Kraus Periodicals Co., Route 100, Millwood, NY 10546.

The Pacific Journal of Mathematics ISSN 0030-8730 is published monthly by the Pacific Journal of Mathematics at P.O. Box 969, Carmel Valley, CA 93924. Application to mail at Second-class postage rates is pending at Carmel Valley, California, and additional mailing offices. Postmaster: Send address changes to Pacific Journal of Mathematics, P. O. Box 969, Carmel Valley, CA 93924.

PUBLISHED BY PACIFIC JOURNAL OF MATHEMATICS, A NON-PROFIT CORPORATION

Copyright $(1983$ by Pacific Journal of Mathematics 


\section{Pacific Journal of Mathematics}

Vol. 108, No. $2 \quad$ April, 1983

Enrique Atencia and Francisco Javier Martin-Reyes, The maximal ergodic Hilbert transform with weights $\ldots \ldots \ldots \ldots \ldots \ldots \ldots \ldots \ldots . \ldots 257$

Bruce Blackadar, The regular representation of local affine motion

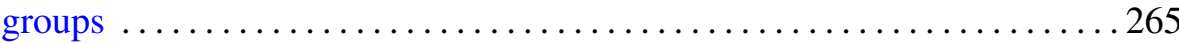

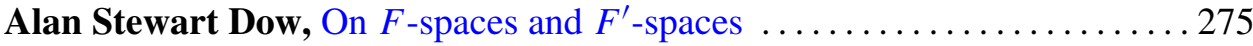

Yoshifumi Kato, On the vector fields on an algebraic homogeneous space . . 285

Dmitry Khavinson, Factorization theorems for different classes of analytic functions in multiply connected domains $\ldots \ldots \ldots \ldots \ldots \ldots \ldots \ldots 295$

Wei-Eihn Kuan, A note on primary powers of a prime ideal . . . . . . . . 319

Benjamin Michael Mann and Edward Yarnell Miller, Characteristic classes for spherical fibrations with fibre-preserving free group

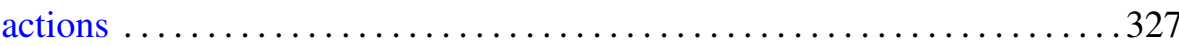

Steven Alan Pax, Appropriate cross-sectionally simple four-cells are flat . . . 379 R. K. Rai, On orthogonal completion of reduced rings ................ 385

V. Sree Hari Rao, On random solutions of Volterra-Fredholm integral

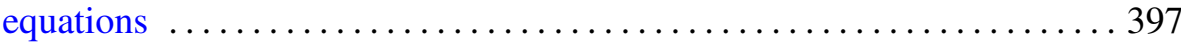

Takeyoshi Satō, Integral comparison theorems for relative Hardy spaces of solutions of the equations $\Delta u=P u$ on a Riemann surface $\ldots \ldots \ldots . .407$

Paul Sydney Selick, A reformulation of the Arf invariant one $\bmod p$ problem and applications to atomic spaces

Roelof Jacobus Stroeker, Reduction of elliptic curves over imaginary quadratic number fields

Jacob Towber, Natural transformations of tensor-products of representation-functors. I. Combinatorial preliminaries

James Chin-Sze Wong and Abdolhamid Riazi, Characterisations of amenable locally compact semigroups 